\title{
SPACELIKE CAPILLARY SURFACES IN THE LORENTZ-MINKOWSKI SPACE
}

\author{
JUNCHEOL PYO and KEOMKYO SEO ${ }^{凶}$
}

(Received 27 October 2010)

\begin{abstract}
For a compact spacelike constant mean curvature surface with nonempty boundary in the threedimensional Lorentz-Minkowski space, we introduce a rotation index of the lines of curvature at the boundary umbilical point, which was developed by Choe ['Sufficient conditions for constant mean curvature surfaces to be round', Math. Ann. 323(1) (2002), 143-156]. Using the concept of the rotation index at the interior and boundary umbilical points and applying the Poincaré-Hopf index formula, we prove that a compact immersed spacelike disk type capillary surface with less than four vertices in a domain of $\mathbb{L}^{3}$ bounded by (spacelike or timelike) totally umbilical surfaces is part of a (spacelike) plane or a hyperbolic plane. Moreover, we prove that the only immersed spacelike disk type capillary surface inside a de Sitter surface in $\mathbb{L}^{3}$ is part of (spacelike) plane or a hyperbolic plane.
\end{abstract}

2010 Mathematics subject classification: primary 53A10; secondary 53C42.

Keywords and phrases: capillary surfaces, spacelike surfaces, constant mean curvature.

\section{Introduction}

Spacelike surfaces with constant mean curvature (CMC) in the three-dimensional Lorentz-Minkowski space have been studied for a long time. Besides the importance of spacelike CMC surfaces in mathematics, such surfaces have played an important role in the study of general relativity (see [6, 15] for a survey). In [2], using integral formulas for compact spacelike CMC surfaces in $\mathbb{L}^{3}$, Alías et al. proved that the only immersed compact spacelike CMC surfaces in $\mathbb{L}^{3}$ spanning a circle are the (spacelike) planar disks and the hyperbolic caps. Moreover, this uniqueness result was generalized to the $n$-dimensional Lorentz-Minkowski space by Alías and Pastor [3]. One year later, Alías and Pastor [4] introduced a variational problem for spacelike surfaces in $\mathbb{L}^{3}$ whose critical points are indeed spacelike CMC surfaces intersecting a given support surface of a constant hyperbolic angle. For these spacelike CMC surfaces with free boundary in $\mathbb{L}^{3}$, they were able to prove the following theorem.

This research was supported by the Basic Science Research Program through the National Research Foundation of Korea (NRF) funded by the Ministry of Education, Science and Technology (20100022951 and 2010-0004246).

(C) 2011 Australian Mathematical Publishing Association Inc. 0004-9727/2011 \$16.00 

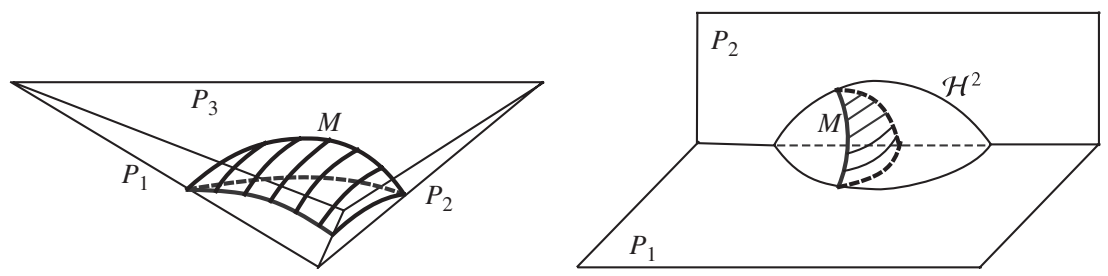

FigURE 1. Spacelike capillary surface $M$ with three vertices inside a domain bounded by: three (spacelike or timelike) planes $P_{i}, i=1,2,3$ (left); a spacelike plane $P_{1}$, a timelike plane $P_{2}$ and a hyperbolic plane $\mathcal{H}^{2}$ (right).

THEOREM [4]. The only immersed spacelike CMC surfaces in $\mathbb{L}^{3}$ with (spacelike) planar or hyperbolic support surfaces are the planar disks $(H=0)$ and the hyperbolic caps $(H \neq 0)$.

On the other hand, analogous problems for CMC surfaces in the Euclidean space concerning planar disks and spherical caps have been studied as well $[1,7,11$, 13, 14, 19]. In particular, it is well known that a capillary disk in a ball of the three-dimensional Euclidean space must be totally umbilical [16, 20]. This is called Nitsche's theorem. Here a capillary surface $M$ in a domain $U$ is a CMC surface which meets $\partial U$ in a constant contact angle along $\partial M \cap \partial U$. Physically capillary surfaces arise as the surface of an incompressible liquid in a container (see [8] and references therein). In 2002, Choe [5] showed that if a compact immersed disk type capillary surface $(H \neq 0)$ in a domain bounded by planes or spheres in $\mathbb{R}^{3}$ has less than four vertices on its boundary, then the surface must be spherical. Because a regular capillary disk has no vertices on its boundary, Choe's result can be thought of as a generalization of Nitsche's theorem. Motivated by this, we investigate a compact immersed spacelike capillary surface with vertices in $\mathbb{L}^{3}$ bounded by (spacelike or timelike) totally umbilical surfaces.

In order to deal with spacelike CMC surfaces with vertices, we introduce a rotation index of the lines of curvature at the boundary umbilical point, which was first studied by Choe [5]. Using the concept of the rotation index at the interior and boundary umbilical points and applying the Poincaré-Hopf index formula, we prove the following theorem.

THEOREM. Let $M \subset \mathbb{L}^{3}$ be a compact spacelike immersed disk type CMC surface which is $C^{2, \alpha}$ up to and including $\partial M$ and whose boundary is $C^{2, \alpha}$ up to and including its vertices. Suppose that each regular component of $\partial M$ is a line of curvature. If the number of vertices of $M$ with angle less than $\pi$ is less than or equal to 3 , then $M$ is part of a (spacelike) plane or a hyperbolic plane.

As a consequence of this theorem, we obtain the following uniqueness theorem (see Figure 1).

THeOREM. Let $U \subset \mathbb{L}^{3}$ be a domain bounded by (spacelike or timelike) totally umbilical surfaces in $\mathbb{L}^{3}$ and let $M$ be a compact spacelike immersed disk type capillary surface 
in $U$ which is $C^{2, \alpha}$ up to and including $\partial M$ and whose boundary is $C^{2, \alpha}$ up to and including its vertices. If $M$ has fewer than four vertices with angle less than $\pi$, then $M$ is part of a (spacelike) plane or a hyperbolic plane.

Our theorems can be regarded as an extension of Alías and Pastor [4], since they showed the uniqueness theorem for regular spacelike capillary surfaces which have no vertices. As another application, we prove that the only immersed spacelike disk type capillary surface inside a de Sitter surface in $\mathbb{L}^{3}$ is part of a (spacelike) plane $(H=0)$ or a hyperbolic plane $(H \neq 0)$. (See Theorem 4.5.)

\section{Preliminaries}

Let $\mathbb{L}^{3}$ be the three-dimensional Lorentz-Minkowski space, that is, the real vector space $\mathbb{R}^{3}$ endowed with the Lorentz-Minkowski metric $\langle$,$\rangle , where \langle\rangle=,d x_{1}{ }^{2}+d x_{2}{ }^{2}-$ $d x_{3}{ }^{2}$ and $x_{1}, x_{2}, x_{3}$ are the canonical coordinates of $\mathbb{R}^{3}$. If $M \subset \mathbb{L}^{3}$ is an embedded connected spacelike surface, we shall denote by $N_{M}$ the unique future-directed unit normal timelike vector field on $M$. Here we call a vector $v \in \mathbb{L}^{3}$ future-directed if $v$ has the same orientation as $(0,0,1) \in \mathbb{L}^{3}$. We say that a vector $v \in \mathbb{L}^{3} \backslash\{0\}$ is spacelike, timelike or lightlike if $|v|^{2}=\langle v, v\rangle$ is positive, negative or zero, respectively. The zero vector 0 is spacelike by convention. A plane in $\mathbb{L}^{3}$ is said to be spacelike or timelike if the normal vector of the plane is timelike or spacelike, respectively. An immersed surface $\Sigma \subset \mathbb{L}^{3}$ is called spacelike if every tangent plane is spacelike. We now give some examples of spacelike and timelike surfaces.

(i) The horizontal plane $\left\{x_{3}=c\right\}$ for a constant $c \in \mathbb{R}$ is spacelike and the vertical plane $\left\{a x_{1}+b x_{2}=0\right\}$ is timelike for any constants $a, b \in \mathbb{R}$ except $a=b=0$.

(ii) The hyperbolic plane

$$
\mathcal{H}^{2}(-c)=\left\{x=\left(x_{1}, x_{2}, x_{3}\right) \in \mathbb{L}^{3}:\langle x, x\rangle=-c^{2}, x_{3}>0\right\}
$$

is a spacelike surface for a positive constant $c \in \mathbb{R}$. The unit normal vector is the position vector itself for each point on the hyperbolic plane.

(iii) The de Sitter surface is defined as

$$
\mathcal{S}^{2}(c)=\left\{x \in \mathbb{L}^{3}:\langle x, x\rangle=c^{2}\right\}
$$

for a positive constant $c \in \mathbb{R}$. Note that the de Sitter surface is timelike and the unit normal vector is also the position vector itself for each point on the de Sitter surface.

Let $M \subset \mathbb{L}^{3}$ be a spacelike or timelike surface. A point $p \in M$ is called umbilical if for any $\xi_{1}, \xi_{2} \in T_{p} M$,

$$
I I_{p}\left(\xi_{1}, \xi_{2}\right)=\lambda(p)\left\langle\xi_{1}, \xi_{2}\right\rangle,
$$

that is, the second fundamental form $I I$ is proportional to the first fundamental form. If the immersion is spacelike, this is equivalent to saying that two principal curvatures are equal at $p$. A surface is called totally umbilical if any point is umbilical. The 
(spacelike or timelike) totally umbilical surfaces in the three-dimensional LorentzMinkowski space are classified as follows.

Theorem 2.1 [17, p. 116]. The only totally umbilical surfaces in $\mathbb{L}^{3}$ are planes, hyperbolic planes and de Sitter surfaces.

Throughout this paper, we shall use two different Lorentzian timelike angles in the three-dimensional Lorentz-Minkowski space in addition to the usual Lorentzian spacelike angle [18]. If $u$ and $v$ are future-directed timelike vectors in $\mathbb{L}^{3}$, then the Lorentzian timelike angle between $u$ and $v$ is defined to be the unique nonnegative real number $\beta(u, v)$ such that

$$
\langle u, v\rangle=|u \| v| \cosh \beta(u, v),
$$

where $|w|$ denotes the absolute value of $\langle w, w\rangle^{1 / 2}$ for a timelike vector $w \in \mathbb{L}^{3}$. In fact, this Lorentzian timelike angle between two timelike vectors was called a hyperbolic angle in [4]. If $u$ is a spacelike vector and $v$ is a future-directed timelike vector in $\mathbb{L}^{3}$, then the Lorentzian timelike angle between $u$ and $v$ is defined to be the unique nonnegative real number $\beta(u, v)$ such that

$$
\langle u, v\rangle=|u \| v| \sinh \beta(u, v) .
$$

For simplicity, we will call $\beta(u, v)$ the angle between $u$ and $v$.

\section{Rotation index}

Let $X: M \hookrightarrow \mathbb{L}^{3}$ be a spacelike immersion. Consider an isothermal coordinate $z=u+i v$ on $M$ taking values in a simply connected domain $\Omega \subset M$. The metric of $\Omega$ induced by the immersion $X$ can be written as $d s^{2}=\lambda^{2}|d z|^{2}$. Since every spacelike surface is orientable, one can define a timelike normal vector field $N$ on $M$ which satisfies $\langle N, N\rangle=-1$. Thus it is easy to see that $\left\{X_{u}, X_{v}, N\right\}$ is an orthogonal frame on $\Omega$ and

$$
\begin{aligned}
& X_{u u}=\frac{\lambda_{u}}{\lambda} X_{u}-\frac{\lambda_{v}}{\lambda} X_{v}-e N, \\
& X_{u v}=\frac{\lambda_{v}}{\lambda} X_{u}+\frac{\lambda_{u}}{\lambda} X_{v}-f N, \\
& X_{v v}=-\frac{\lambda_{u}}{\lambda} X_{u}+\frac{\lambda_{v}}{\lambda} X_{v}-g N,
\end{aligned}
$$

where

$$
\begin{aligned}
& e=-\left\langle N_{u}, X_{u}\right\rangle=\left\langle N, X_{u u}\right\rangle, \\
& f=-\left\langle N_{u}, X_{v}\right\rangle=\left\langle N, X_{u v}\right\rangle=-\left\langle N_{v}, X_{u}\right\rangle, \\
& g=-\left\langle N_{v}, X_{v}\right\rangle=\left\langle N, X_{v v}\right\rangle .
\end{aligned}
$$

If we put $\Phi(z, \bar{z})=e-g-2 i f$, the Codazzi equation implies that the Hopf function $\Phi(z, \bar{z})$ is holomorphic with respect to the complex coordinate $z$ if and only if the 
immersion $X$ is a spacelike CMC immersion. It is well known that $\Phi d z^{2}$ is a holomorphic quadratic differential.

Since the principal curvature $\kappa$ and the infinitesimal principal vector $\left(\begin{array}{c}d u \\ d v\end{array}\right)$ satisfy $d N\left(\begin{array}{l}d u \\ d v\end{array}\right)=\kappa\left(\begin{array}{l}d u \\ d v\end{array}\right)$, one can see that the equation for the lines of curvature is given by

$$
-f d u^{2}+(e-g) d u d v+f d v^{2}=0,
$$

which implies, for $z=u+i v$, that

$$
\operatorname{Im}\left(\Phi d z^{2}\right)=0
$$

This is equivalent to

$$
\arg \Phi+2 \arg (d z)=m \pi \quad(m \text { an integer })
$$

or

$$
\arg (d z)=\frac{m \pi}{2}-\frac{1}{2} \arg \Phi
$$

The lines of curvature of a spacelike surface generate a smooth line field except at umbilical points. They rotate sharply around the umbilical points. Note that the umbilical points are isolated because such points are the zeros of the holomorphic function $\Phi$. The rotation index of the lines of curvature at an umbilical point is defined as

$$
I=\frac{1}{2 \pi} \delta(\arg d z)=-\frac{1}{4 \pi} \delta(\arg \Phi),
$$

where $\delta$ denotes the variation if one goes once around an isolated umbilical point. Therefore if the umbilical point $p$ is in the interior of the spacelike surface, then $p$ is a zero of $\Phi$ of order $n(\geq 1)$ and $\delta(\arg \Phi)=2 \pi n$. Thus at an interior point $p$ we have

$$
I(p)=-\frac{n}{2} \leq-\frac{1}{2}
$$

So far we have discussed the rotation index of the lines of curvature at the interior umbilical points. From now on, we consider the case where the umbilical point $q$ is on the boundary of the spacelike surface. In [5], Choe introduced the rotation index of the lines of curvature at a boundary umbilical point $q$. Using his idea, we can estimate the rotation index. We briefly discuss the definition of the rotation index at the boundary umbilical point. We may assume that a neighborhood of $q$ is a conformal immersion of a half disk $D_{h}=\left\{(u, v) \in \mathbb{R}^{2}: u^{2}+v^{2}<1, v \geq 0\right\}, X: D_{h} \rightarrow M \subset \mathbb{L}^{3}$ with the diameter $l$ of $D_{h}$ into the boundary of the spacelike surface and $X(0)=q$. Since $X(l)$ is a line of curvature of $M$, this line field can be extended smoothly to a line field $L$ on the whole disk $D=\left\{(u, v) \in \mathbb{R}^{2}: u^{2}+v^{2}<1\right\}$ by reflection through the diameter. So one can define the rotation index of the lines of curvature at the boundary umbilical point $q$ to be half the rotation index of $L$ at 0 . This is independent of the choice of the immersion $X$. Thus at a boundary umbilical point $q$ such that $\Phi$ has a zero of order $n$, one sees that

$$
I(q)=\frac{1}{2}\left[-\frac{1}{4 \pi} \delta(\arg \Phi)\right]=-\frac{n}{4}
$$


A singular point of the boundary $\partial M$ of a spacelike surface $M \subset \mathbb{L}^{3}$ is called a vertex. For an immersed CMC surface in $\mathbb{R}^{3}$, Choe gave an estimate for the rotation index at the boundary umbilical points and vertices. Since the rotation index is intrinsically defined, we shall make use of his results without proof.

Lemma 3.1 [5]. Let $M \subset \mathbb{L}^{3}$ be an immersed spacelike CMC surface which is $C^{2, \alpha}$ up to and including $\partial M$ and whose boundary is $C^{2, \alpha}$ up to and including its vertices. If the smooth components of $\partial M$ are lines of curvature, then the following properties hold.

(a) The boundary umbilical points of $M$ are isolated.

(b) At a boundary umbilical point which is not a vertex of $M$ the rotation index of lines of curvature is not greater than $-1 / 4$.

(c) At a vertex of $M$ with angle less than $\pi$, the rotation index is less than or equal to $1 / 4$, and at a vertex with angle greater than $\pi$, the rotation index is less than or equal to $-1 / 4$.

Lemma 3.2 [5]. Assume that $M$ and $\partial M$ are the same as in Lemma 3.1 and assume that $p$ is a vertex of $M$ with angle $\xi$. If $\xi<\pi$ and $p$ is a singularity of $\Phi$, then $p$ is a simple pole. If $\xi>\pi$, then $p$ is a zero of $\Phi$.

From the above rotation index estimate, we can prove the following uniqueness theorem for an immersed spacelike CMC surface in $\mathbb{L}^{3}$. The proof is based on [5].

THEOREM 3.3. Let $M \subset \mathbb{L}^{3}$ be a compact immersed spacelike disk type CMC surface which is $C^{2, \alpha}$ up to and including $\partial M$ and whose boundary is $C^{2, \alpha}$ up to and including its vertices. Suppose that each regular component of $\partial M$ is a line of curvature. If the number of vertices of $M$ with angle less than $\pi$ is less than or equal to three, then $M$ is part of a (spacelike) plane or a hyperbolic plane.

Proof. The well-known Poincaré-Hopf theorem [9] says that the sum of the rotation indices of all singularities of a vector field is equal to the Euler characteristic of the surface. Therefore one sees that if $V$ is a line field on the domain $D$ with a finite number of singularities which is the pull-back under $x: D \rightarrow M$ of the lines of curvature on $M$, then the sum of the rotation indices of $V$ at the singularities in $\bar{D}$ is equal to 1 . So $M$ has a nonempty set $S$ of singularities. Moreover, the singularities of the lines of curvature on $M$ occur not only at the umbilical points but also at the vertices of $M$. Here the umbilical points correspond to the zeros of $\Phi$ and the vertices correspond to the poles or zeros of $\Phi$ by Lemma 3.2.

Now suppose the singular set $S$ is finite. Let $p_{i}, q_{j}, r_{k}$ and $s_{l}$ be the interior umbilical points, nonvertex boundary umbilical points, vertices with angle greater than $\pi$ and vertices with angle less than $\pi$, respectively. Then from inequality (3.1) and Lemma 3.1 it follows that

$$
\begin{aligned}
\sum_{p=p_{i}, q_{j}, r_{k}, s_{l}} I(p) & \leq \sum_{i}\left(-\frac{1}{2}\right)+\sum_{j}\left(-\frac{1}{4}\right)+\sum_{k}\left(-\frac{1}{4}\right)+\sum_{l}\left(\frac{1}{4}\right) \\
& \leq \sum_{l}\left(\frac{1}{4}\right) \\
& \leq \frac{3}{4} \text { (by hypothesis) }
\end{aligned}
$$


which contradicts the fact that $\sum I(p)=1$ from the Poincaré-Hopf theorem. Hence one deduces that $S$ is infinite and has an accumulation point $q$. Furthermore, if $\kappa_{1}$ and $\kappa_{2}$ are principal curvatures of $M$, then $S$ is the zero set of the continuous function $\kappa_{1}-\kappa_{2}$, and hence $q \in S$ and $S$ is closed. However, the points of $S$, except the vertices with angle less than $\pi$, are also the zeros of $\Phi$. Since the zero set of the holomorphic function $\Phi$ is either open or finite, it follows that $S=M$, and therefore $M$ is totally umbilical. Thus one can conclude that $M$ is part of a (spacelike) plane or a hyperbolic plane.

\section{Spacelike capillary surfaces}

Consider a domain $U \subset \mathbb{L}^{3}$ whose boundary $\partial U$ is a piecewise embedded connected spacelike or timelike surface. A spacelike capillary surface $M$ in a domain $U \subset \mathbb{L}^{3}$ is an immersed spacelike CMC surface which meets $\partial U$ at a constant contact angle along $\partial M$. If $\partial U$ is a piecewise smooth surface, then we may assume the constant angles to be distinct on each smooth component of $\partial U$.

Let $M \subset \mathbb{L}^{3}$ be a spacelike capillary surface which meets $\partial U$ at a constant contact angle $\beta$. We denote by $\tau$ the positively oriented unit tangent vector field along $\partial M$ and denote by $N$ the timelike unit normal vector field on $M$. Clearly $\{\tau, N, v=-\tau \wedge N\}$ is trihedral along $\partial M$. Here $u \wedge v$ denotes the vector product of two vectors $u, v \in \mathbb{L}^{3}$ which is defined to be the unique vector $u \wedge v \in \mathbb{L}^{3}$ such that

$$
\langle u \wedge v, w\rangle=\operatorname{det}(u, v, w)
$$

for any $w \in \mathbb{L}^{3}$ [2]. Clearly $v=-\tau \wedge N$ is the inward-pointing unit conormal vector field along $\partial M$. Choose a regular piece $\Sigma$ of $\partial U$. Then $\Sigma$ is an embedded connected spacelike or timelike surface. As before, one may construct trihedra $\left\{\tau, N_{\Sigma}, v_{\Sigma}\right\}$ along $\partial M$, where $N_{\Sigma}$ is the unit normal vector field on $\Sigma$ and $v_{\Sigma}$ is the inward-pointing unit conormal vector field along $\partial \Sigma$ which is given by $v=-\tau \wedge N_{\Sigma}$. For these two trihedra $\{\tau, N, v\}$ and $\left\{\tau, N_{\Sigma}, v_{\Sigma}\right\}$, we have the following equations.

(i) If $\Sigma$ is a spacelike surface,

$$
\left\{\begin{array}{l}
v=\cosh \beta v_{\Sigma}+\sinh \beta N_{\Sigma} \\
N=\sinh \beta v_{\Sigma}+\cosh \beta N_{\Sigma}
\end{array}\right.
$$

(ii) If $\Sigma$ is a timelike surface,

$$
\left\{\begin{array}{l}
v=\sinh \beta v_{\Sigma}+\cosh \beta N_{\Sigma} \\
N=\cosh \beta v_{\Sigma}+\sinh \beta N_{\Sigma}
\end{array}\right.
$$

In cases where $\Sigma$ is a spacelike surface, Alías and Pastor [4] also used Equation (4.1) in which the constant contact angle $\beta$ only differs by a minus sign from ours.

When the ambient space is a Euclidean space, the following Terquem-Joachimsthal theorem is well known.

THEOREM [21]. Let c be a curve in $M_{1} \cap M_{2} \subset \mathbb{R}^{3}$ which is a line of curvature in $M_{1}$. Then $c$ is a line of curvature in $M_{2}$ if and only if $M_{1}$ and $M_{2}$ intersect at a constant contact angle along $c$. 
This theorem can be generalized to the three-dimensional Lorentz-Minkowski space as follows. It should be mentioned that Alías and Pastor [4] proved this lemma for a spacelike surface $\Sigma$. The method we use here is a modification of [4]. For the sake of completeness we give the proof.

LEMMA 4.1. Let $M \subset \mathbb{L}^{3}$ be an immersed spacelike CMC surface and let $\Sigma \subset \mathbb{L}^{3}$ be a (spacelike or timelike) totally umbilical surface. Suppose that $M$ meets $\Sigma$ at a constant contact angle along $\partial M \cap \Sigma$. Then each smooth component of $\partial M \cap \Sigma$ is a line of curvature of $M$.

Proof. Choose a point $p$ on a smooth component of $\partial M \cap \Sigma$. It suffices to show that the intersection of a local neighborhood of $p$ with $\partial M \cap \Sigma$ is a line of curvature of $M$. Let $X: D_{h} \rightarrow M \subset \mathbb{L}^{3}$ be a conformal immersion of a half disk

$$
D_{h}=\left\{(u, v) \in \mathbb{R}^{2}: u^{2}+v^{2}<1, v \geq 0\right\}
$$

into $M$, which maps the diameter $l$ of $D_{h}$ into $\partial M$ and $X(0)=p$. Let $z=u+i v$ be the usual coordinates in $\mathbb{C}$. Then the metric on $M$ is written by $d s^{2}=\lambda^{2}|d z|^{2}$ for a positive smooth function $\lambda=\lambda(z)$. One can write the unit tangent vector field $\tau$ and the inward-pointing unit conormal vector field $v$ along the smooth boundary containing $p$ as $\tau=\lambda^{-1} \partial_{u}$ and $v=\lambda^{-1} \partial_{v}$. By Theorem 2.1, we have four possible cases for $\Sigma$ : a spacelike plane, a hyperbolic plane, a timelike plane and a de Sitter surface.

When $\Sigma$ is a spacelike plane, the normal vector field of $\Sigma$ is given by $N_{\Sigma}=\vec{a}$,

$$
\begin{aligned}
I I(\tau, v) & =-\left\langle\bar{\nabla}_{\tau} N, v\right\rangle=\left\langle N, \bar{\nabla}_{\tau} v\right\rangle \\
& =\cosh \beta\left\langle\bar{\nabla}_{\tau} v_{\Sigma}, N\right\rangle+\sinh \beta\left\langle\bar{\nabla}_{\tau} \vec{a}, N\right\rangle \\
& =\cosh \beta \sinh \beta\left\langle\bar{\nabla}_{\tau} v_{\Sigma}, v_{\Sigma}\right\rangle+\cosh ^{2} \beta\left\langle\bar{\nabla}_{\tau} v_{\Sigma}, \vec{a}\right\rangle \\
& =\frac{1}{2} \cosh \beta \sinh \beta \tau\left\langle v_{\Sigma}, v_{\Sigma}\right\rangle-\cosh ^{2} \beta\left\langle v_{\Sigma}, \bar{\nabla}_{\tau} \vec{a}\right\rangle \\
& =0,
\end{aligned}
$$

where $\bar{\nabla}$ denotes the connection of $\mathbb{L}^{3}$.

When $\Sigma$ is a de Sitter surface, the normal vector field of $\Sigma$ is given by $N_{\Sigma}=X$,

$$
\begin{aligned}
I I(\tau, v) & =-\left\langle\bar{\nabla}_{\tau} N, v\right\rangle=\left\langle N, \bar{\nabla}_{\tau} v\right\rangle \\
& =\sinh \beta\left\langle\bar{\nabla}_{\tau} v_{\Sigma}, N\right\rangle+\cosh \beta\left\langle\bar{\nabla}_{\tau} X, N\right\rangle \\
& =\cosh \beta \sinh \beta\left\langle\bar{\nabla}_{\tau} v_{\Sigma}, v_{\Sigma}\right\rangle+\sinh ^{2} \beta\left\langle\bar{\nabla}_{\tau} v_{\Sigma}, X\right\rangle \\
& =\frac{1}{2} \cosh \beta \sinh \beta \tau\left\langle v_{\Sigma}, v_{\Sigma}\right\rangle-\sinh ^{2} \beta\left\langle v_{\Sigma}, \tau\right\rangle \\
& =0 .
\end{aligned}
$$

When $\Sigma$ is a hyperbolic or timelike plane, the proof is similar to the case where $\Sigma$ is a spacelike plane or a de Sitter surface.

THeOREM 4.2. Let $U \subset \mathbb{L}^{3}$ be a domain bounded by (spacelike or timelike) totally umbilical surfaces in $\mathbb{L}^{3}$ and let $M$ be a compact immersed spacelike disk type 
capillary surface in $U$ which is $C^{2, \alpha}$ up to and including $\partial M$ and whose boundary is $C^{2, \alpha}$ up to and including its vertices. If $M$ has less than four vertices with angle less than $\pi$, then $M$ is part of a (spacelike) plane or a hyperbolic plane.

Proof. From Lemma 4.1, we obtain that each smooth component of $\partial M$ is a line of curvature of $M$. Hence the conclusion follows from Theorem 3.3.

REMARK 4.3. In the case where a smooth component of $\partial U$ is a lightlike plane, one cannot expect a similar equation to (4.1) or (4.2) since $N$ is a lightlike vector. Thus the proof of Theorem 4.2 does not work in this case.

REMARK 4.4. The number of vertices of $M$ with angle less than $\pi$ in Theorem 4.2 is sharp. Let $C$ be the Lorentzian catenoid which is a spacelike surface of revolution [10, 12]. Consider $M \subset C$ to be a compact part bounded by two parallel horizontal spacelike planes which are perpendicular to the axis of $C$ and two vertical timelike planes containing the axis of $C$ with angle $\theta \in(0, \pi)$. Then $M$ is a compact embedded disk type spacelike CMC $(H=0)$ capillary surface with four vertices with angle less than $\pi$ at each of which the rotation index equals $1 / 4$, which is part of neither a (spacelike) plane nor a hyperbolic plane.

As an immediate consequence of Theorem 4.2, one can obtain the following theorem which is a generalization of [4].

THEOREM 4.5. The only spacelike immersed disk type capillary surface inside a de Sitter surface in $\mathbb{L}^{3}$ is a planar disk $(H=0)$ or a hyperbolic disk $(H \neq 0)$.

\section{References}

[1] L. Alías, R. López and B. Palmer, 'Stable constant mean curvature surfaces with circular boundary', Proc. Amer. Math. Soc. 127(4) (1999), 1195-1200.

[2] L. Alías, R. López and J. Pastor, 'Compact spacelike surfaces with constant mean curvature in the Lorentz-Minkowski 3-space', Tohoku Math. J. 50 (1998), 491-501.

[3] L. Alías and J. Pastor, 'Constant mean curvature spacelike hypersurfaces with spherical boundary in the Lorentz-Minkowski space', J. Geom. Phys. 28(1-2) (1998), 85-93.

[4] L. Alías and J. Pastor, 'Spacelike surfaces of constant mean curvature with free boundary in the Minkowski space’, Classical Quantum Gravity 16 (1999), 1323-1331.

[5] J. Choe, 'Sufficient conditions for constant mean curvature surfaces to be round', Math. Ann. 323(1) (2002), 143-156.

[6] Y. Choquet-Bruhat and J. York, The Cauchy Problem. General Relativity and Gravitation, Vol. 1 (Plenum, New York, 1980), pp. 99-172.

[7] R. Earp, F. Brito, W. Meeks and H. Rosenberg, 'Structure theorems for constant mean curvature surfaces bounded by a planar curve', Indiana Univ. Math. J. 40(1) (1991), 333-343.

[8] R. Finn, Equilibrium Capillary Surfaces, Grundlehren der Mathematischen Wissenschaften, 284 (Springer, New York, 1986).

[9] H. Hopf, Differential Geometry in the Large, Lecture Notes in Mathematics, 1000 (Springer, Berlin, 1989).

[10] O. Kobayashi, 'Maximal surfaces in the 3-dimensional Minkowski space $L^{3}$, , Tokyo J. Math. 6(2) (1983), 297-309.

[11] M. Koiso, 'Symmetry of hypersurfaces of constant mean curvature with symmetric boundary', Math. Z. 191(4) (1986), 567-574. 
[12] F. López, R. López and R. Souam, 'Maximal surfaces of Riemann type in Lorentz-Minkowski space $\mathbb{L}^{3}$ ', Michigan Math. J. 47(3) (2000), 469-497.

[13] R. López and S. Montiel, 'Constant mean curvature discs with bounded area', Proc. Amer. Math. Soc. 123(5) (1995), 1555-1558.

[14] R. López and S. Montiel, 'Constant mean curvature surfaces with planar boundary', Duke Math. J. 85(3) (1996), 583-604.

[15] J. Marsden and F. Tipler, 'Maximal hypersurfaces and foliations of constant mean curvature in general relativity', Phys. Rep. 66(3) (1980), 109-139.

[16] J. Nitsche, 'Stationary partitioning of convex bodies', Arch. Ration. Mech. Anal. 89(1) (1985), $1-19$.

[17] B. O'Neill, Semi-Riemannian Geometry with Application to Relativity, Pure and Applied Mathematics, 130 (Academic Press, New York, 1983).

[18] J. Ratcliffe, Foundations of Hyperbolic Manifolds, 2nd edn, Graduate Texts in Mathematics, 149 (Springer, New York, 2006).

[19] A. Ros and H. Rosenberg, 'Constant mean curvature surfaces in a half-space of $R^{3}$ with boundary in the boundary of the half-space', J. Differential Geom. 44(4) (1996), 807-817.

[20] A. Ros and R. Souam, 'On stability of capillary surfaces in a ball', Pacific J. Math. 178(2) (1997), $345-361$.

[21] M. Spivak, A Comprehensive Introduction to Differential Geometry, Vol. III (Publish or Perish, Berkeley, CA, 1979).

JUNCHEOL PYO, Department of Mathematics, Pusan National University, Busan 609-735, Korea

e-mail: juncheolpyo@gmail.com

KEOMKYO SEO, Department of Mathematics, Sookmyung Women's University, Hyochangwongil 52, Yongsan-ku, Seoul 140-742, Korea

e-mail: kseo@sookmyung.ac.kr 